UDC 621.928 .2

A. D. BARDOVSKIY ${ }^{1}$, Professor, Doctor of Engineering Sciences

S. M. GORBATYUK ${ }^{1}$, Head of Process Equipment Engineering Department, Professor, Doctor of Engineering Sciences, sgor02@mail.ru

A. A. GERASIMOVA ${ }^{1}$, Associate Professor, Candidate of Engineering Sciences

I. I. BASYROV ${ }^{1}$, Assistant at Process Equipment Engineering Department

${ }^{1}$ College of Environmentally Sound Technologies and Engineering, NUST MISIS, Moscow, Russia

\title{
ANALYSIS OF OPERATION FEATURES OF SIZING SCREEN WITH PARAMETRIC EXCITATION
}

\section{Introduction}

Commercial separation of mineral fines is complicated by chocking of screen meshes with wet or 'difficult' grains, which greatly reduces the sifting surface and, consequently, worsens the sieve sizing efficiency [1, 2]. Prevention of such unwanted difficulties during sieving of wet granular materials by means of creation of favorable conditions for selfcleaning of sifting surface can enable major improvements in sieve sizing efficiency.

Existing experience of sizing difficult-tosieve and wet granular materials in Russia and abroad shows that a promising solution to this problem can be the separation screens with vibrating sifting surfaces. The review of the designs of vibrating screens and the research of their separation efficiency revealed their major advantages as against conventiona shaking screens. The advantages include compound motion and deformation of the sifting surface, high rate of loosening and mixing of a screenable material, activation of the sieve self-cleaning owing to higher relative movability of the sizing screen components, reduced energy input and material content of the screen, as well as weaker impact on the footing of a separation screen [3-6].

All these advantages belong in the separation screen with parametric excitation of sifting surface. This type screen differs from the screens with direct excitation of sifting surface by simpler design and by resonance vibrations of the sieve. Transferability of tension and flexure deformations to an operating sieve ensures efficient self-cleaning of the sieve openings from adhered material and stuck grains. The wave motion of the sifting surface supposes the use of sieves made of elastic materials (rubber, polyurethane) which perceive alternating load and possess high abrasion resistance. Small sifting surface is balanced by high movability of the sieve components as compared with the steel braided sieves widely applied in screen sizing $[7,8]$.

The studies of the parametric resonances in engineering mainly focused on recommendations on the resonance elimination to enhance reliability of machines. Regarding screen sizing, the parametric resonance is used with intent as a beneficial effect, which governs a new route of the research.

Free screen sizing is amply discussed [9-15], while the behavior of difficult-to-sift particles on a sifting surface with parametric excitation remains yet poorly studied.

Structurally, a separation screen with parametric excitation of the sifting surface is a screen with direct excitation [16-22].
Lateral vibrations of the sieve are excited by means of change in the flexural stiffness of its elastic components by applying the periodic axial force on them (in plane of the sieve) at a frequency two times higher than the free frequency of the sifting surface. The vibration mode is governed by the half-wave

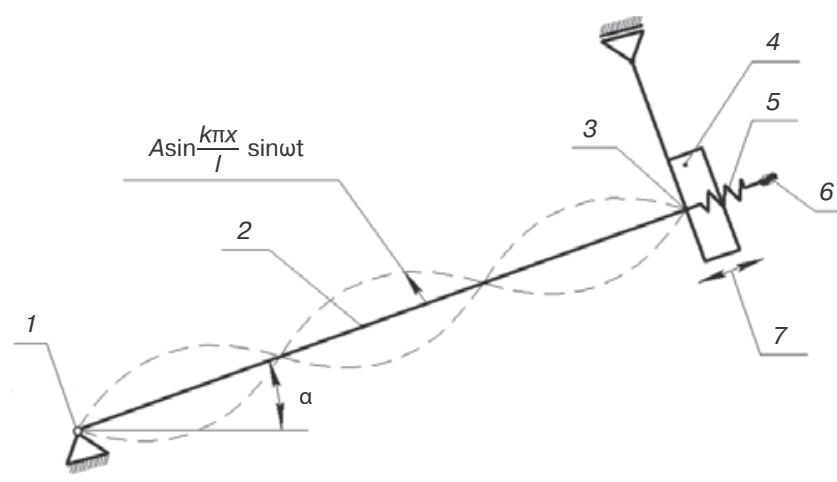

Fig. 1. General layout of screener with parametric excitation of sifting surface:

1 - fixed mounting of screen (hinge mount); 2 - sieve; 3 - movable mounting of screen; 4 - directed-action vibration exciter; 5 spring linkage; 6 - stretching device; 7 - travel direction of vibration exciter 
number $K$ of lateral vibrations, which depends on the screen geometrics and manner of fastening, as well as on the value of process load.

Motion of the sifting surface is described with a nonlinear differential equation:

$$
\frac{d^{2} A}{d t^{2}}+\frac{2 \varepsilon d A}{d t}+\omega_{k}^{2}(1-\mu \cos \theta t) A+\psi\left(A ; \frac{d A}{d t} ; \frac{d^{2} A}{d t^{2}}\right)=0
$$

where $A$ is the amplitude of oscillations of the sieve; $\omega_{k}$ is the frequency of the sieve vibrations of a K-th form governed by the half-wave number of lateral oscillations of the sieve; $\mu=$ $=p_{t} / p_{0}$ is the coefficient of pulsation; $\varepsilon$ is the oscillation attenuation coefficient; $\psi\left(A ; d A / d t ; d^{2} A / d t^{2}\right)$ is a nonlinear function; $\theta$ is the sieved material viscosity.

\section{Basic procedures and results of the research}

The behavior of a nonlinear function depends on the factors of nonlinear attenuation, nonlinear inertia and nonlinear elasticity. The latter is the dominating nonlinear factor in the system under analysis. With regard to this factor, solution of (1) comes to the relation for finding the amplitude of steady-state lateral oscillations of the sieve:

$$
A=\frac{2 \omega_{k}}{(3 \gamma)^{1 / 2}}\left[n^{2}-1 \pm\left(\frac{\mu^{2}}{4}-\frac{n^{2} \Delta^{2}}{\pi^{2}}\right)^{1 / 2}\right]^{1 / 2}
$$

In expression (2), $\gamma$ and $n$ are the values found in terms of the parameter $K$, reduced stiffness $C_{r}$ of the system, sieve vibration attenuation decrement $\Delta$, as well as the mass and geometrics of the sieve.

For a screen with parametric excitation, it is typical that the particle flow experiences vibrations and constant tossing. As a result, after each toss, a particle falls down, and it is probable that the particle passes through a sieve opening. This probability can be given by:

$$
p=\frac{\left(d \sin a_{f}-D\right)(c-D)}{(d+e) \sin a_{f}(c+e)},
$$

where $d$ and $c$ are the areal sizes of a mesh, $\mathrm{mm}$; $D$ is the diameter of a particle, $\mathrm{mm}$; $e$ is the strand width, $\mathrm{mm}$; $a_{\mathrm{f}}$ is the angle of particle fall on the sieve, deg.

Considering that the particle jump probability is $q=1-p$, the probability of the particle pass through the sieve at an $n$-th point of the sieve at the preset fidelity $\varepsilon$ is of the form of:

$p_{\mathrm{n}}=q_{1} \cdot q_{2} \cdot \ldots \cdot q_{n-1} \cdot p_{n}<\varepsilon$

Relation (4) makes it possible to find the required length of the sifting surface for particle sizing at the preset fidelity $\varepsilon$ : $I_{m p}=\Sigma I_{i}$, where $I_{i}$ is the projection of a particle fall path on the sieve center line at an $i$-th stage of the particle flight.

Motion of a particle at the flight stage is described by a system of differential equations below:

$$
\left\{\begin{array}{l}
m d^{2} x / d t^{2}=m g \sin a \\
m d^{2} y / d t^{2}=-m g \cos a
\end{array}\right.
$$

The flight initiation condition is the zero normal force $N$ found from the system of equations describing the particle flow on the curved sieve surface:

$$
\left\{\begin{array}{l}
m\left(v^{2} / \rho\right)=N-m g \cos (\alpha+\beta) \\
m(d v / d t)=f N-m g \sin (\alpha+\beta)
\end{array}\right.
$$

where $a$ is the incline of the screen box; $\beta$ is the angle between the tangent to the sieve curvature and the screen box center line; $\rho$ is the sieve curvature radius at the test point.

Separation efficiency of difficult-to-sieve granular materials greatly depends on relative mobility of the sifting surface components as it creates favorable conditions for self-cleaning of the sifting surface from adhered materials and particles stuck in the mesh. For mesh sieves, the relative mobility of the sieve components is achieved by imparting one or a few modes of deformation to them. The main deformation modes in the screens with direct excitation are tension, bending and shearing. For a screen with parametric excitation, the typical deformation modes are tension and bending. Tension is conditioned by the periodic axial force effective in the plane of the sieve. The bending deformation is connected with the wave motion of the sieve, when cross sections of the sieve rotate relative one another.

The sieve length under parametric vibration is given by:

$$
\mathrm{S}_{(t)}=b^{-1}\left(1+p^{2}\right)^{1 / 2} E\left[k \pi, p\left(1+p^{2}\right)^{-1 / 2}\right],
$$

where $b$ and $p$ are some coefficients found from parameters of the vibration process; $E\left[k \pi, p\left(1+p^{2}\right)^{-1 / 2}\right]$ is the second-order elliptic integral.

The time-dependent sizes of the sieve mesh, conditioned by tension are

$$
\begin{aligned}
& d_{p(t)}=d_{0}\left(1+\varepsilon_{p(t)}\right), \\
& c_{p(t)}=c_{0}+e \mu_{f} \varepsilon_{p(t)},
\end{aligned}
$$

where $d_{0}$ and $c_{0}$ are the mesh sizes in the longitudinal and lateral directions, respectively; $\mu_{1}$ is the lateral deformation coefficient of the sieve material (Poisson's ratio); $\varepsilon_{p(t) \text {, }}$ is the relative deformation of the sieve during vibration.

The mutual rotation angle of cross sections in normal direction to the screen center line is equal to the angle of contingence of tangents to the sieve bend line at the test points. Considering deformation of tension and bending, the timedependent sizes of the mesh and the internal angle between the opposite mesh walls are:

$$
\begin{aligned}
& d_{(x . t)}=d_{0}\left(1+\varepsilon_{p(t)}\right)+r_{B} \Delta \delta_{(x . t)} ; \\
& c_{(t)}=c_{0}+e \mu_{f} \varepsilon_{p(t)} ; \\
& \delta_{(x . t)}=\delta_{0}+\Delta \delta_{(x . t)},
\end{aligned}
$$

where $\Delta \delta_{(x . t)}$ is the change in the angle between the opposite mesh walls during parametric vibrations.

The analysis of the sieve deformation shows that the relative deformation value linearly grows with an increasing angle of shear between the longitudinal and lateral vibrations, and reaches a peak value at the phase shift angle of $\pi / 2$.

A limiting size grain stuck in a mesh experiences forces from the mesh walls, as well as gravity and inertia. In unfavorable conditions (friction or gripping between the particle and mesh walls exceed the expulsive force), the particle can stay in the mesh for a long time and can prevent passing of other particles thereby. A particle can go from the mesh with oversize fraction or with undersize fraction. In this respect, the mesh emptying condition and removal of the stuck grain can be given by: 


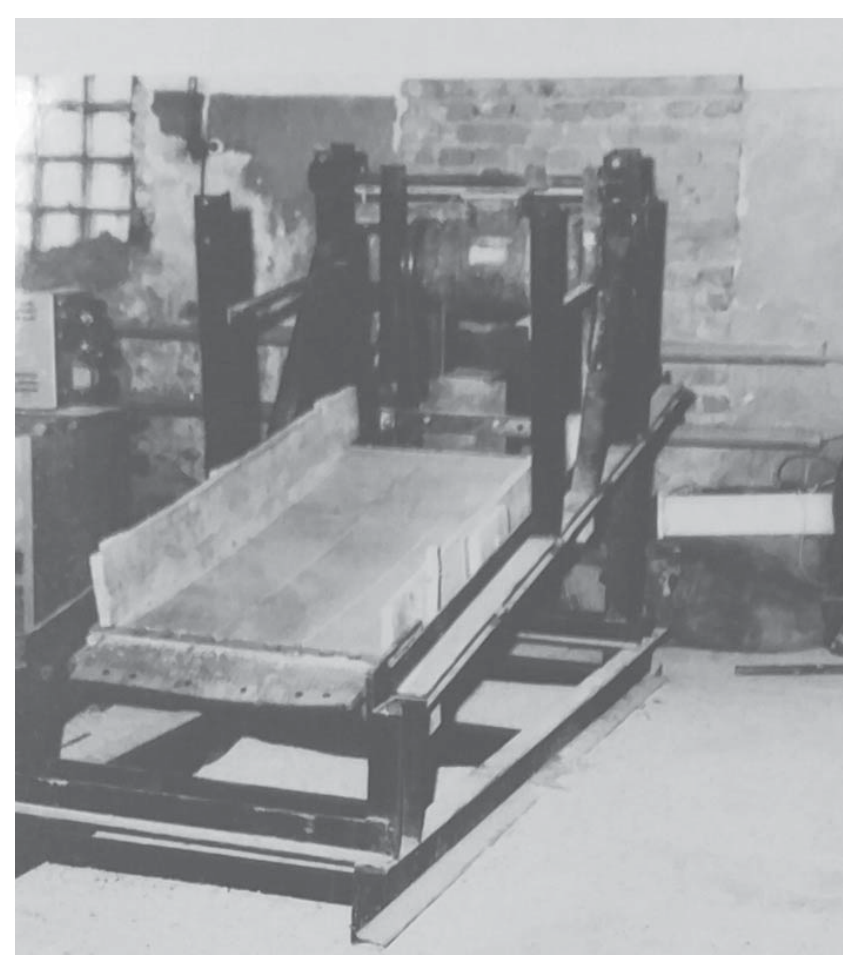

Fig. 2. General view of sizing screen test bench with mechanical excitation of sieve

- when particle goes with oversize fraction:

$m y^{\prime \prime} \geq m g+2 N \sin \left(\Delta \delta_{(x . t)} / 2\right)+2 f N \cos \left(\Delta \delta_{(x . t)} / 2\right)$

- when particle goes with undersize fraction:

$$
m y^{\prime \prime}+m g+2 N \sin \left(\Delta \delta_{(x . t)} / 2\right) \geq 2 f N \cos \left(\Delta \delta_{(x . t)} / 2\right)
$$

A more favorable condition of separation is when a particle goes with undersize fraction as then the particle never comes in the mesh and the repeated jamming is eliminated.

For the sizing tests of wet limestone aggregates $5 \mathrm{~mm}$ in size, a sizing screen test bench with parametric excitation of the sifting surface was designed and manufactured. The sifting surface was a rubber rolled sieve with mesh size $4.5 \times 10$ $\mathrm{mm}$. The sieve design and selection of operation conditions are described in [23, 24].

The general view of the sizing screen test bench is shown in Fig. 2, and deformation of the sieve under two half-waves of vibration is demonstrated in Fig. $\mathbf{3}$.

The test scope embraced motion paths of points of the sifting surface under vibration in the mode of parametric resonance. Such vibrations typically have paths of the type of a Lissajous figures at a frequency ratio of longitudinal and lateral vibrations of $2: 1$.

When the sieve enters the mode of parametric resonance, the paths of the points acquire a crescent shape which then transforms into a serpentine path due to the phase shift between the longitudinal and lateral vibrations. At the phase shift angle of $\pi / 2$, quenching of the lateral vibrations take place, and the sieve enters the mode of forced vibrations at a frequency ratio of longitudinal and lateral vibrations of 1:1.

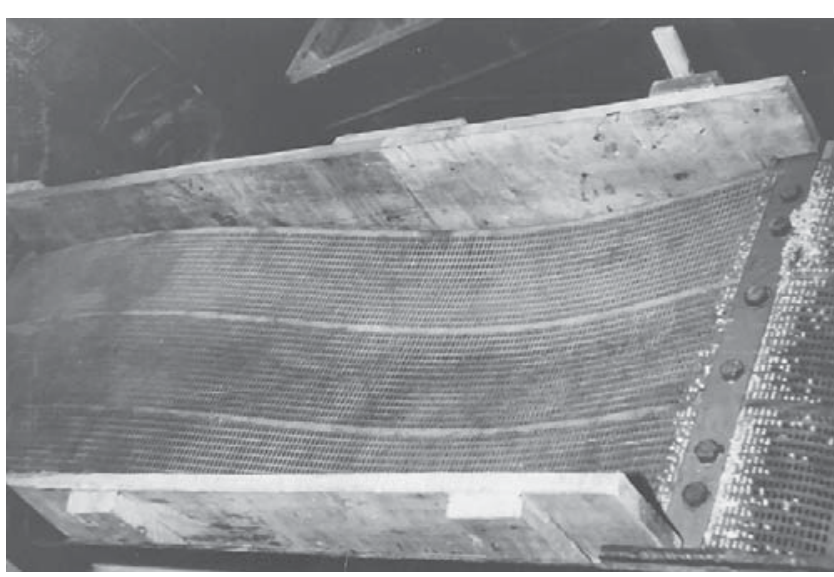

Fig. 3. Deformation of sieve under two half-waves of vibrations

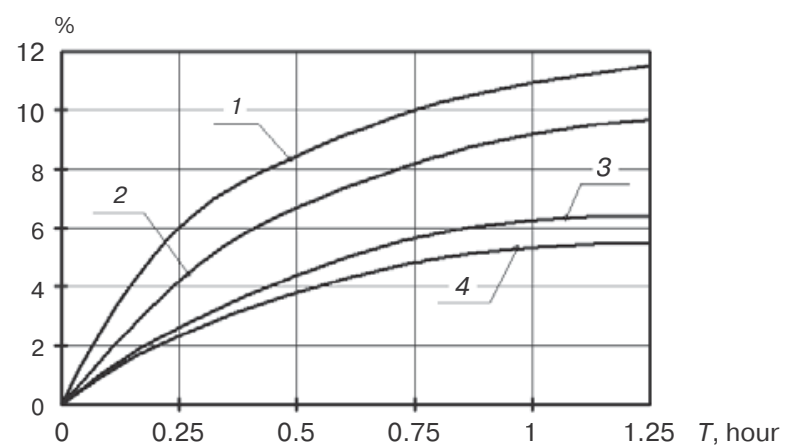

Fig. 4. Screening surface loss versus time of screen sizing in different operation conditions:

$1-K=1 ; 2-K=2 ; 3-K=3 ; 4-K=4$

The presence of the lateral vibrations is explained by the sag of the sieve under own weight and the weight of the load.

In the tests, we determined the separation efficiency of wet carbonate aggregates versus the sieve design and operation conditions. The carbonate aggregates had a size of $-10 \mathrm{~mm}$ at the $-5 \mathrm{~mm}$ size content of $44.6 \%$. The moisture content of the aggregates was maintained at the level of $10-12 \%$, which represented the most unfavorable sizing conditions.

Due to small weight of the sieve and owing to the resonant vibration mode, separation on the sieve with parametric excitation is possible under small loads which ensure steady-state lateral vibrations. Such load is not to exceed $2 \mathrm{t} /\left(\mathrm{h} \cdot \mathrm{m}^{2}\right)$ at the exciter vibration amplitude of $5 \mathrm{~mm}$ and four half-waves of the sieve vibrations.

Classification of the undersize sampled at different points along the sifting surface to analyze the grain size distribution shows that the content of flaky grains in the undersize grows at the final stage of screening, in the zone nearby the immobile frame of the sieve. This is explained by the lower flow velocity in this zone and by the buildup of the particle layer thickness as a consequence of reduced amplitudes of the longitudinal and lateral vibrations of the sieve at the immobile frame, which ends with extrusion of liming size grains through the mesh under the overlying material weight.

In the estimate of the sifting surface self-cleaning efficiency, we determined the screening surface loss versus the 
sieve operation parameters and the screen sizing time. It is found that the screening surface loss grows nonlinearly for a certain time after the sieving start, and the open space of the screen is stabilized then (Fig. 4).

Actually, in the beginning of the screen sizing processes, the sifting surface is chocked at the test points with limiting size grains due to insufficient value of mutual displacement of the opposite mesh walls. Further, the mesh jamming and emptying come to equilibrium and the value of the open space of the screen gets stabilized.

The comparative analysis of the testing data shows that during size screening of fine and wet carbonate aggregates, the rate of self-cleaning of the sieve meshes is 1.5 times higher in case of the sifting surface with parametric excitation than with conventional-type vibro-screens in the same operation conditions. Positively, the sieves with parametric excitation are more efficient in sizing of wet fines of difficult-to-sieve materials or materials with high content of grains of the same size as the sieve meshes have in case of low specific load applied to the sieves, i.e., in field conditions.

\section{Conclusions}

1. Parametric excitation of the sifting surface of a sizing screen ensures sizing of difficult-to-screen mineral fines owing to integrated effect of longitudinal and lateral vibrations of the sifting surface components and due to deformation of the sieve meshes in vibration.

2. The value of relative deformation under parametric vibrations grows linearly with an increasing angle of phase shift between the longitudinal and lateral vibrations and achieves the maximum at the phase shift angle of $\pi / 2$.

3. In the beginning of size screening, the sifting surface is chocked with limiting size grains at the test points due to insufficient mutual displacement of the opposite mesh walls. Then the value of the open space of the screen is stabilized.

4. The sizing screens with parametric excitation are the most efficient in sizing of fine and wet difficult-to-screen materials at small process loads on the sieve.

\section{References}

1. Morozov V. I. Estimate of sifting surfaces of shaking screens for dry sizing of nonmetal waste with natural moisture content at open pit mines. GIAB. 1998. No. 4. pp. 49-54.

2. Bardovskii A. D., Gerasimova A. A., Keropyan A. M., Bibikow P. Ya. Influence of the mechanical characteristics of harp screen material on screening process. Izvestiya vuzov, Chernaya metallurgiya. 2018. No. 9(1). pp. 678-682. DOI: 10.17073/0368-0797-2018-9-678-682.

3. Starikovskiy P. P. Design validation of sizing screens with parametric excitation for wet carbonate material separation. Candidate of Engineering Sciences Dissertation. Moscow : MGI, 1989. $147 \mathrm{p}$.

4. Kharitonov A. O., Bibikov P. Ya., Verzhanskiy P. M., Voronin B. V. Classification of pressing machines for separation of weak rocks. Modern scientific knowledge: theory, methodology, practice : Collected papers. Smolensk, 2016. pp. 72-73.

5. Sivachenko L. A., Dobrovolsky Yu. K. History of development of grinding engineering and its up-to-date level. Vestnik Belorussko-Rossiyskogo universiteta. 2012. No. 4(37). pp. 69-76.

6. Bibikov P. Ya., Bardovskiy A. D., Mitusov P. E., Kalakutskiy A. V. Design in addition shredder classifier for processing weak rocks. GIAB. 2015. No 8. pp. 166-170.

7. Bardovsky A. D., Gerasimova A. A. Analysis of drive mechanism of side-to-side moving flexible blade. GIAB. 2019 No. 7. pp. 132-139. DOI: 10.25018/0236-1493-2019-07-0132-139.

8. Shapoval A. N., Shapoval A. A. Development of the unit for multi-stage vibration drawing of metal products. Tsvetnye Metally. 2002. No. 4. pp. 77-82.

9. Kartavyy N. G. Grinding and sizing equipment for waste processing at open pit nonmetal mines. Nauchnye shkoly MGGU. 2008. Vol. 2. pp. 318-325.

10. Naumova M., Basyrov I., Aliev K. Reengineering of the ore preparation production process in the context of Almalyk MMC JSC. MATEC Web of Conferences. 2018. 01030. DOI: $10.1051 /$ matecconf/201822401030

11. Bratan S., Roshchupkin S. Synthesis of lunberger stochastic observer for estimation of the grinding operation state. MATEC Web of Conferences. 2018. Vol. 224. 01133. DOI: 10.1051/ matecconf/201822401133.

12. Lyashenko V. I., Dyatchin V. Z., Franchuk V. P. Engineering and commissioning of vibrating screeners-feeders for the mining industry. Chernaya metallurgiya. 2014. No. 5. pp. 72-80.

13. Mamonov S. V., Tsipin E. F., Bratygin E. V. Conditions of self cleaning of the screen surface for thin hydraulic screening. Izvestiya vuzov. Gornyy zhurnal. 2014. No. 5. pp. 106-111.

14. Fomin A. A., Gusev V. G., Sattarova Z. G. Geometrical errors of surfaces milled with convex and concave profile tools. Solid State Phenomena. 2018. Vol. 284. pp 281-288.

15. Bardovsky A. D., Gerasimova A. A., Basyrov I. I. Study of Oscillating Process of Harp Screens. Lecture Notes in Mechanical Engineering. Springer. 2019. pp 133-139.

16. Gorbatyuk S. M., KeropyanA. M., Bibikov P. Ya. Assessing Parameters of the Accelerator Disk of a Centrifugal Mill Taking into Account Features of Particle Motion on the Disk Surface. Journal of Friction and Wear. 2018. Vol. 39, No. 4. pp. 326-329.

17. Jain C. V., Panchve D. P. Design and development of Cam operated multiple vibrating screening machine. International Journal of Emerging Technology and Advanced Engineering. 2016. Vol. 6, Iss. 2. pp. 78-80.

18. Wills B. A., Finch J. A. Wills' mineral processing technology. Elsevier, 2015. $512 \mathrm{p}$.

19. Zhang B., Zhu G., Lv B., Yan G. A novel and effective method for coal slime reduction of thermal coal processing. Journal of Cleaner Production. 2018. Vol. 198. pp. 19-23.

20. Gupta A., Yan D. Mineral processing design and operations. An introduction. 2nd ed. Elsevier, 2016. 882 p.

21. Roshchupkin S., Kharchenko A. Method of building dynamic relations, estimating product and grinding circle shape deviations. MATEC Web of Conferences. 2018. Vol. 224. 01001. DOI: 10.1051/matecconf/201822401001.

22. Slobodyanik T. M., Balakhnina E. E. Dynamics of elementary differential composed of elastic bodies. GIAB. 2019. No. 9 . pp. 204-210.

23. Starikovskiy P. P. Amplitudes of parametric vibrations of screeners made of elastic materials. Equipment for Integrated Use of Mineral Products : Collected Papers. Moscow, 1987. pp. 67-71.

24. Kartavyy N. G., Starikovskiy P. P., Pushpakbaev B. T., Kafanov L. S. Separation of granular materials on sieves with parametric excitation. Promyshlennost stroitelnykh materialov Moskvy. 1986. No. 4. pp. 11-14. 